\title{
The Role of Small Redundant Actuators in Precise Manipulation
}

\author{
Ravi Balasubramanian* and Yoky Matsuoka
}

\begin{abstract}
With the goal of developing human-like dextrous manipulation, we investigate how the central nervous system uses the redundant control space of the human hand to perform tasks with force-stiffness requirements. Specifically, while the human hand is actuated by several muscles with varying mechanical advantage (called the moment arm), it is unclear how each muscle is used. Using the Anatomically Correct Testbed (ACT) robotic hand to compute the control solution space and human-subject experiments with surface electromyography to measure biological control strategy, we identified that there is significant redundancy in the control spaces of both muscles with large moment arms and muscles with small moment arms. However, the central nervous system was selective about the solution for muscles with large moment arms, while it chose to span large regions of the available control space for muscles with small moment arms. Furthermore, the biological solution used low-moment-arm muscles at relatively high actuation levels. We summarize by making inferences on why the central nervous system chooses such a strategy and how this can help robotic manipulation.
\end{abstract}

\section{INTRODUCTION}

Several hand mechanisms have been designed over the years, but it has been difficult to replicate the human hand's dexterity. The human hand is equipped with intricate biomechanics and neural control which make possible a variety of end-effector grasps and dynamic movements. We believe that understanding the features of the human hand and how the central nervous system exploits them can contribute significantly to improving robotic manipulation. Toward this goal, this paper explores the redundant control space of the human hand using an anatomically correct robotic hand and humansubject experiments.

Inspired by the human hand, our group has developed the Anatomically Correct Testbed (ACT) hand [26], [5], which carefully mimics the human hand's actuation, tendon structure, and bone shapes (see Fig 1). The goals of building the ACT hand are several: the ACT

R. Balasubramanian and Y. Matsuoka are with Department of Computer Science and Engineering University of Washington, WA.

*Contact author: bravi@Cs. washington. edu

This work was partially funded by NIH R01NS050256.

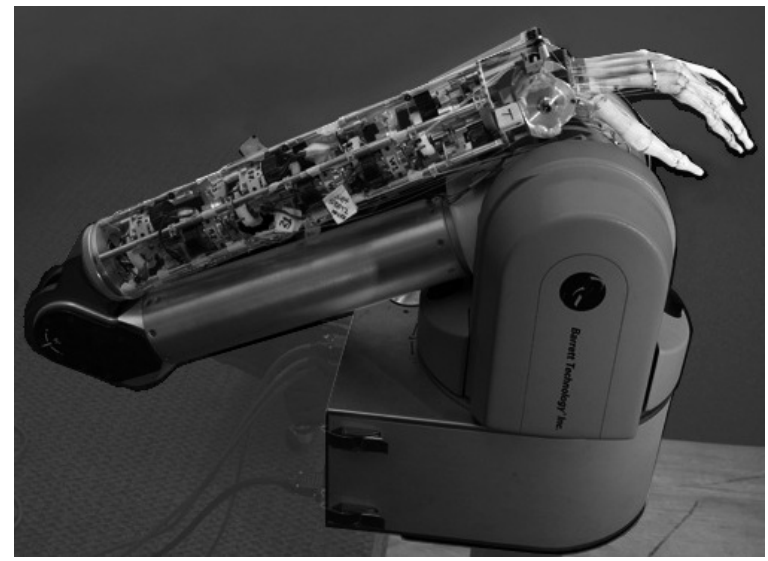

Fig. 1. The Anatomically Correct Testbed (ACT) robotic hand has five fingers (three are fully actuated) and preserves the human musculotendon structure.

hand is a vehicle for 1) physiologists to study the passive and active biomechanics of the human hand, 2) neuroscientists to investigate the neural signal coding to enable functional hand movement, 3) surgeons to test reconstruction techniques for impaired hands, and 4) roboticists to build better telemanipulation tools including a prosthetic hand.

Our system identification of the ACT hand [9] has identified that the complex tendon routing and bone shapes play a significant role in producing critical postures or forces. What has not been investigated so far is the role of redundant muscle arrangement in the human hand. Redundancy allows stiffness modulation for maintaining stability, and the importance of this ability has been identified both in the robotics community [13], [19] and in the neuroscience community [1], [25], [15], [16], [6]. However, such stiffness control could be achieved through simpler redundant arrangements, for example, by having two opposing muscles per joint. Understanding the specific role the human hand's redundant muscle arrangement plays in manipulation will help us build future robotic and prosthetic devices.

The human hand is controlled by many muscles, some in the forearm (called extrinsic muscles) and some in the hand itself (called intrinsic muscles). For example, the index finger has seven muscles, four extrinsic and three intrinsic. These seven muscles control the finger's four degrees of freedom through a complex 
web of tendons creating a redundant control space. Dictated by the effective moment arms, each muscle's influence on hand motion varies with the movement and posture. For example, intrinsic muscles have smaller moment arms for finger flexion (curling) motion than the extrinsic muscles, or when extrinsic muscles are used for abduction and adduction (side-to-side) motion. However, it is not clear how moment arms influence the control solution spaces. As we build an anatomically correct robotic system for biological investigation or as a prosthetic device, it would be critical to understand how the central nervous system (or the controller) utilizes intrinsic and extrinsic muscles differently for fine dextrous control.

It has been noticed that the intrinsic muscles contribute to maintaining grasp stability and fingertip force direction, and that the extrinsics provide actuation for free motion [18]. Also, the role of intrinsic muscles in free motion, power grips, and precision grips have also been identified [14]. To date, the level of investigation of intrinsic and extrinsic muscle utilization presented in this paper has not been done utilizing a robotics framework. With the robotics framework, we can visualize the complete redundant control solution space to produce specific end-effector force, stiffness, and posture. Once the redundant space is explicitly mapped, the structure of the space and the optimization strategies can be discussed. This paper studies an index finger at a specific common posture as an example, and the control strategy found is generalizable to other postures and fingers.

The structure of this paper is as follows: first, we present the framework we use to study the manipulation problem; second, we present experimental results from human-subject experiments; and finally, we discuss the implication of human strategies in the robotic framework to understand the role of redundancy toward manipulation dexterity.

\section{FRAMEWORK FOR MANIPULATION ANALYSIS}

This section presents a framework for finding the control solution space for a manipulation task specifying a force-stiffness combination in a posture. As an example, we selected a scenario where the left index finger is producing flexion (pushing down) forces at two stiffness levels (low and high), and we will use the ACT index finger to model the human finger. The ACT index finger is built with six muscles, three extrinsic and three intrinsic muscles. In contrast, humans have seven muscles where two of the extrinsic muscles act exactly in the same way to the index finger. The only difference is that one of them also controls other fingers at the same time. Thus for our analysis where we are studying the index finger in isolation, it is sufficiently anatomical to study six muscles. Three components of the framework are: A) the finger's moment arm structure, B) muscle force and stiffness parameters, C) a method to understand the finger's control spaces.

\section{A. Biomechanical Structure: Moment Arms}

The relationship between muscle actuation and joint motion may be expressed in kinematic space as

$$
i=R \dot{\theta},
$$

where $l \in \mathbb{R}^{6}$ represents the muscle lengths, $\theta \in \mathbb{R}^{4}$ the finger's four degrees of freedom (side-to-side and three curling joints), and $R$ the moment arm matrix. We use the following angle convention: curling and towardthe-pinky movements are positive. The influence of the moment arm matrix extends to the dynamic space also, expressed as

$$
\tau=R^{T} f_{m},
$$

where $\tau$ represents the joint torques and $f_{m} \geq 0$ the muscle forces (pulling defined positive).

The moment arm parameters cannot be directly measured from the human subjects, and there is limited data available from cadavers [2]. Our ACT hand moment arm information [9] is the first full data (all joints and muscles) available, and thus we choose to use the ACT moment arms for our analysis. Since we restrict our analysis to only one posture (joint angles from knuckle to tip: 45,45 , and 10 degrees), the moment arm matrix is fixed (see values in Table I). We note that the extrinsic muscles (top three rows) play a greater role in flexion and extension of the finger (second, third, and fourth columns), while the intrinsic muscles (bottom three rows) play a greater role in side-to-side movements (first column).

\section{B. Muscle Activation and Muscle Stiffness}

For each muscle, muscle activation $\alpha$ is defined as the fraction of the maximum muscle force $f_{m, \max }$ used under static and non-fatigued conditions [11]:

$$
\alpha=f_{m} / f_{m, \max } .
$$

Muscle activation provides a way to measure the use of each muscle in a normalized manner, and permits a comparison of the neural signals each muscle receives from the central nervous system. Note that each muscle has a different maximum force and estimates vary (see 
TABLE I

ACT Hand Moment-Arm Matrix For Curled Posture And Maximum Muscle Forces

\begin{tabular}{|c|c|c|c|c|c|c|}
\hline \multirow{4}{*}{\multicolumn{2}{|c|}{ Muscles }} & \multicolumn{4}{|c|}{ Moment arm $(\mathrm{mm} / \mathrm{rad})$} & \multirow{4}{*}{$\begin{array}{l}\text { Maximum } \\
\text { muscle } \\
\text { force }(\mathrm{N})\end{array}$} \\
\hline & & \multirow{3}{*}{ Side-to-side } & & oints & & \\
\hline & & & \multicolumn{3}{|c|}{ Curling } & \\
\hline & & & Knuckle & Middle & Tip & \\
\hline \multirow{3}{*}{ Extrinsic } & EI & -0.04 & 11.11 & 1.86 & -0.99 & 48.2 \\
\hline & FDP & -0.84 & -10.68 & -7.03 & -3.03 & 65.1 \\
\hline & FDS & -0.14 & -10.83 & -6.87 & 0.12 & 48.2 \\
\hline \multirow{3}{*}{ Intrinsic } & PI & -8.23 & -0.79 & -0.22 & 0.73 & 31.3 \\
\hline & LUM & 9.47 & -2.00 & 0.18 & 0.01 & 4.8 \\
\hline & RI & 6.78 & -9.32 & -1.44 & 3.76 & 77.1 \\
\hline
\end{tabular}

Mean moment arm $\mathrm{sd}=0.68 \mathrm{~mm} / \mathrm{rad}$. Appendix A has the muscle names.

Table I, [10], [20]). All solution spaces in this paper are interpreted in muscle activation space.

We model muscle stiffness $k_{m}$ as a non-linear function of muscle activation $\alpha$ :

$$
k_{m}(\alpha)=k_{\max }\left(1-e^{-\lambda \alpha}\right),
$$

where $k_{\max }=72000 \mathrm{~N} / \mathrm{m}$ and $\lambda=4$ are constants [4], [22], [7], [12]. We also assume muscle stiffnesses are independent of each other (similar to [22]), although this is an open question.

\section{Iso-Torque, Iso-Stiffness, and Iso-effector Spaces}

In order to describe the redundant space available for the neural signals or controller, we view the control solutions in muscle activation space for tasks defined by end-effector force and stiffness. We view the muscle solution space in three ways: the iso-torque space, the iso-stiffness space, and the iso-effector space. These spaces have been defined before in [1] and are described below.

The iso-torque space $\Gamma_{t}$ is the set of muscle activation solutions that produce a specific end-effector force and may be defined as follows:

$$
\Gamma_{t}=\left\{\alpha:\left\|R^{-T} f_{m}-J^{T} f_{e}\right\|^{2}<\epsilon\right\},
$$

where $J$ is the finger Jacobian [8], $f_{e} \in \mathbb{R}^{3}$ the end-effector force, and $\epsilon$ a measure of the allowed error ( $\epsilon$ was set as $5 \%$ of target force in this paper). Due to its mathematical structure, the iso-torque space is a two-dimensional surface (six muscles forces and four joint torque specifications). However, factoring task performance noise, the iso-torque space may be represented as a convex hull. Fig. 2a shows four isotorque spaces viewed in extrinsic muscle space. We note that the solution space decreases in size as endeffector force increases.

The iso-stiffness space $\Gamma_{k}$ is the set of muscle activation solutions that produce a specific end-effector stiffness and may be defined as follows:

$$
\Gamma_{k}=\left\{\alpha:\left\|R^{T} K_{m}\left(f_{m}\right) R-J^{T} K_{e} J\right\|^{2}<\epsilon\right\},
$$

where $K_{m}\left(f_{m}\right) \in \mathbb{R}^{6 \times 6}$ is the muscle stiffness matrix function and $K_{e} \in \mathbb{R}^{3 \times 3}$ the desired end-effector stiffness. The iso-stiffness space is a non-linear space, because of the exponential relationship between muscle force and stiffness. Fig. 2b shows two iso-stiffness surfaces for specific end-effector stiffness perpendicular to the fingerpad. Note that the end-effector stiffness varies as the square of moment arm.

The iso-effector space $\Gamma_{e}$ is the set of muscle activation solutions that produce a specific end-effector force and stiffness combination and is defined as the intersection of the iso-torque and iso-stiffness spaces:

$$
\Gamma_{e}=\Gamma_{t} \bigcap \Gamma_{k} .
$$

Fig. 2c shows an iso-effector surface. The key point with this section is that even when end-effector force, stiffness, and posture are specified, the control space still has redundancy.

\section{Problem Statement}

With several muscles actuating the index finger, the central nervous system needs to choose solutions from a redundant control space to satisfy tasks with forcestiffness requirements (see section II-C). Given the structure of the redundant space and the biomechanical structure, we address the following questions.

First, we want to evaluate the redundant space available for intrinsic and extrinsic muscles separately. Given their differences in the moment arms and maximum forces, we want to identify the space available for the controller to use. Second, given such a structure, we evaluate the biological solutions used and how much of the available control space is actually being used. If the entire space is utilized, the central nervous system may be treating the control space as an uncontrolled manifold [21] or as a control knob with low gains. But if the biological solution is using a small range of available space, then there may be additional optimization criteria used to narrow down to a specific solution. Finally, we are interested in understanding the 


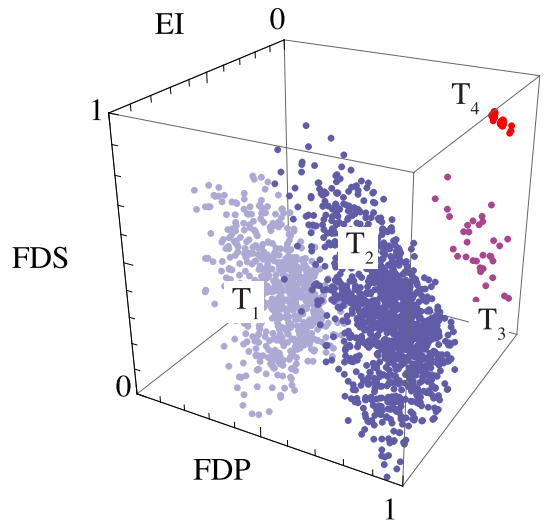

(a)

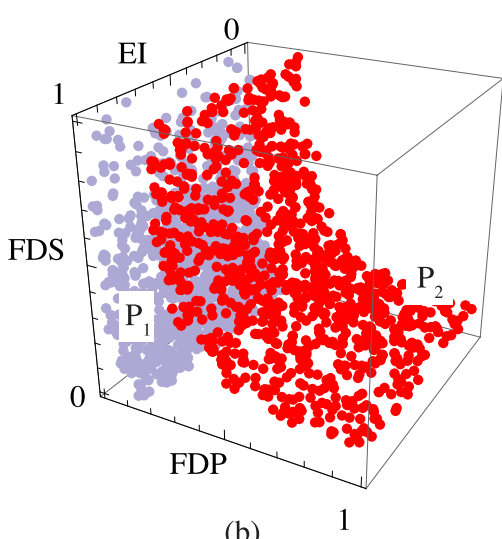

(b)

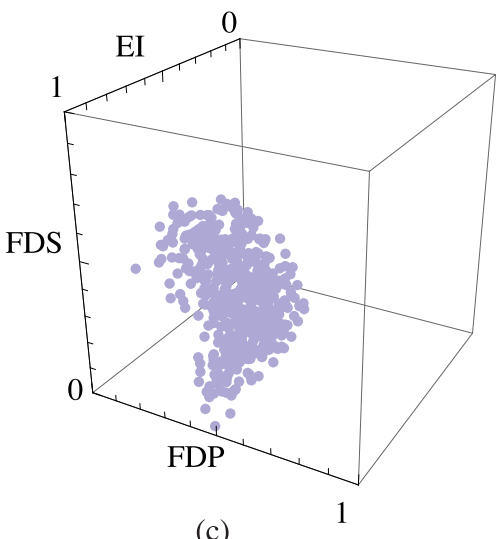

(c)

Fig. 2. (a) Four iso-torque planes with end-effector forces $2 \mathrm{~N}\left(T_{1}\right), 6 \mathrm{~N}\left(T_{2}\right), 10 \mathrm{~N}\left(T_{3}\right)$, and $14 \mathrm{~N}\left(T_{4}\right)$. (b) Two iso-stiffness surfaces with end-effector stiffness in the range 528 to $672 \mathrm{~N} / \mathrm{m}\left(P_{1}\right)$ and 1372 to $1428 \mathrm{~N} / \mathrm{m}\left(P_{2}\right)$. (c) The iso-effector space formed by intersecting the $T_{1}$ with $P_{2}$.

implication of biological usage of extrinsic and intrinsic muscles toward robotic design and controls.

\section{AnAlysis}

\section{A. Comparison of Extrinsic and Intrinsic Muscle Con- trol Spaces}

Fig. 3 shows the iso-torque spaces for two different end-effector forces computed using the ACT hand model. An important aspect of the iso-torque space is that end-effector stiffness varies across the plane. For example, end-effector stiffness (perpendicular to the fingerpad) across iso-torque space in Fig. 3a varies between 238 and $1265 \mathrm{~N} / \mathrm{m}$, and the range matches well with human fingertip stiffness reported in [18] (the target fingertip forces are larger in [18] though). For the purpose of visualization, we split each iso-torque space into two regions (using the midpoint of the stiffness range as threshold), a low stiffness region (blue) and a high stiffness region (red). While the solution space is cleanly divided into two stiffness regions in the extrinsic muscle space, the low and high stiffness solution spaces overlap in the intrinsic muscle space. Furthermore, for a specified end-effector force accuracy requirement, the allowable variability for intrinsic muscle forces for a flexion task is greater than the allowable variability for extrinsic muscle forces. For example, with a permitted error of $5 \%$ on end-effector force, the variability for extrinsic forces is less than $15 \%$ of the available range, while the variability for intrinsic forces is greater than $60 \%$.

\section{B. Human Experimental Procedure}

To assess the biological strategy, human subject experiments were conducted. Four subjects were asked
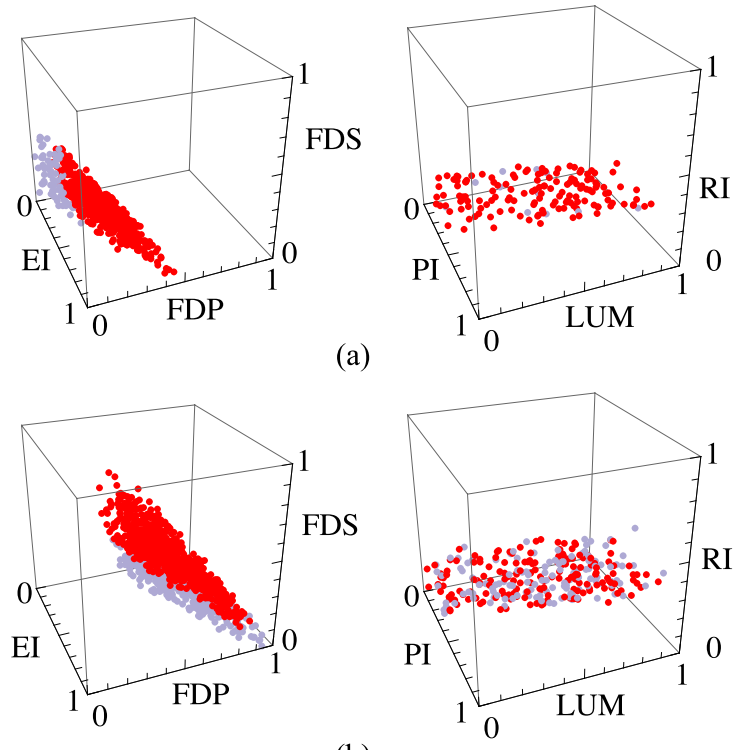

(b)

Fig. 3. The iso-torque planes for two end-effector forces: (a) $2 \mathrm{~N}$, and (b) $10 \mathrm{~N}$. The blue region represents a low-stiffness solution space and the red region a high-stiffness solution space.

to maintain a specific right index finger posture (45, 45, 10 degrees at the knuckle, middle, and tip joints) while a force sensor was placed between their finger pad and a hard surface. In each trial, they were instructed to press against the force sensor at $30 \%$ of their comfortable maximum voluntary force (which was typically between 2 and $3 \mathrm{~N}$ ) with visual feedback for about three seconds. Simultaneously, the subjects were instructed to also hold a specific low or high stiffness value according to their stiffness calibration procedure (where they defined their own comfortable low and high stiffness levels). Surface electromyographic data was collected from one intrinsic muscle (RI) and three extrinsic muscles (EI, FDS, and FDP). We note that 
other muscles are more challenging to access through surface electromyography. The experiment protocol was approved by the University of Washington Human Subjects Division.

\section{Biological Extrinsic and Intrinsic Muscle Solutions}

Table II presents mean and variability of RI muscle activation, expressed as a ratio of the extrinsic flexor and extensor muscle activation, for low and high stiffness levels. We notice that RI's muscle activation mean and variation are significantly larger than the mean and variation of extrinsic muscle activation. Also, the fingertip stiffness maintained by the subjects, estimated using the ACT hand model, is included in Table II and compares well with the fingertip stiffness reported in [18] (the target fingertip forces are larger in [18] though).

\section{Mapping Biological Solutions to Theoretical Space}

Fig. 4 overlays human subject data onto the extrinsic muscle-activation convex hull of the iso-torque space $T_{1}$ in Fig 2. The red blobs indicate the highstiffness solutions (with their standard deviation as the radius) and the green blobs indicate the low-stiffness solutions. As expected, the high-stiffness solution has a greater muscle activation when compared with the low-stiffness solution. We notice that the biological solutions lie in the region of the convex hull.

Fig. 5 shows human subject data overlaid on to two iso-effector spaces (computed using the ACT hand model) viewed in the extrinsic muscle activation space. While the force sensor was used to verify that endeffector force was $2 \mathrm{~N}$, stiffness on the other hand had to be inferred from the biological solution. Specifically, we used the mean activation of the extrinsic muscle solution to compute end-effector stiffness. The first isoeffector space's (blue region) stiffness varied between $528 \mathrm{~N} / \mathrm{m}$ and $672 \mathrm{~N} / \mathrm{m}$ and the second iso-effector space's (red region) stiffness varied between $880 \mathrm{~N} / \mathrm{m}$ and $1120 \mathrm{~N} / \mathrm{m}$. The high-stiffness biological solution volume was about $9 \%$ of the red iso-effector space volume, and low-stiffness biological solution about $8 \%$ relative of the blue iso-effector space volume.

Fig. 6 is equivalent to Fig. 5 for the intrinsic muscle RI. The light color region indicates the iso-effector space available for this specific task, and the dark color indicates the biological solution used with its standard deviation as its width. For our experiment, we only recorded from RI, so we can make the comparison only in a single dimensional space. We notice that the

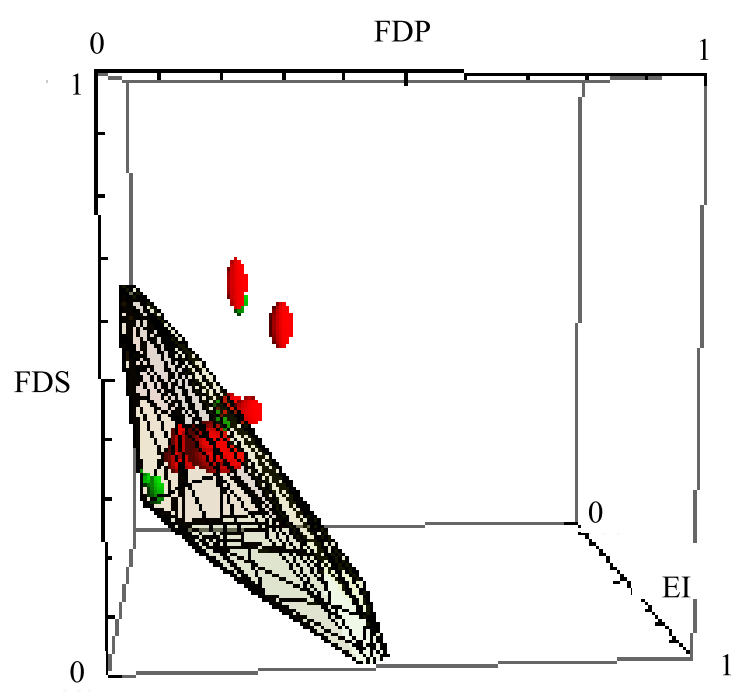

Fig. 4. Extrinsic muscle activation data from three subjects overlaid on the iso-torque space $T_{1}$ in Fig 2. The target end-effector force was $2 \mathrm{~N}$. The green blobs represent the low-stiffness biological solution and the red region the high-stiffness biological solution.

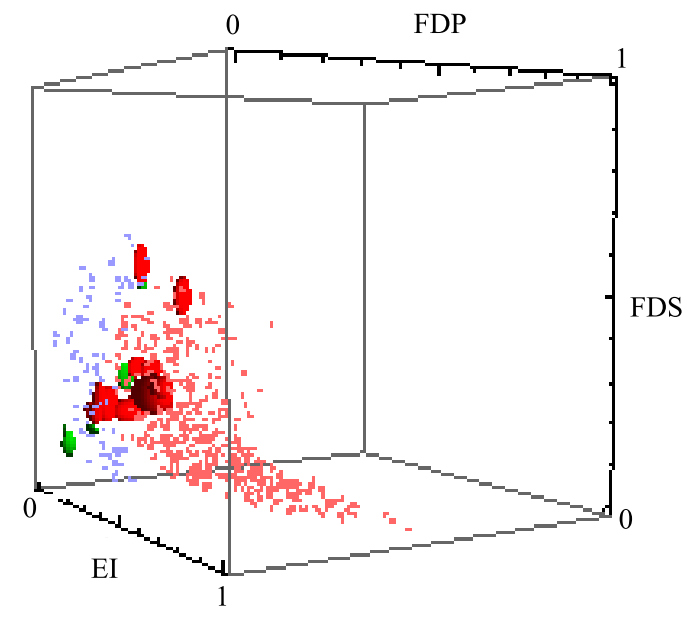

Fig. 5. Extrinsic muscle activation data from three subjects overlaid on two iso-effector surfaces generated using the ACT hand model. The target end-effector force was $2 \mathrm{~N}$. The blue region represents a low-stiffness $(528$ to $672 \mathrm{~N} / \mathrm{m}$ ) solution space and the red region a high-stiffness solution space ( 880 to $1120 \mathrm{~N} / \mathrm{m}$ ). The green blobs represent the low-stiffness biological solution and the red region the high-stiffness biological solution.

biological solution is at the upper end of the available solution space, and variability is large. It was noticed that the RI muscle activation spanned about $36 \%$ of the available control space at low stiffness and $94 \%$ of the available control space at high stiffness.

\section{DISCUSSION}

\section{A. Difference in Extrinsic and Intrinsic control space}

From the ACT hand model, we notice a marked difference in the control solution spaces for intrinsic 
TABLE II

Intrinsic Muscle Activation Compared with Extrinsic Muscle ACtivation

\begin{tabular}{c|c|c|c|c}
\hline Fingertip Stiffness (N/m) & \multicolumn{2}{|c|}{ Mean } & \multicolumn{2}{c}{ Variation } \\
& Intrinsic/Flexor Ratio & Intrinsic/Extensor Ratio & Intrinsic/Flexor Ratio & Intrinsic/Extensor Ratio \\
\hline \hline Low $[237,804]$ & $1.55(0.48)$ & $2.41(0.36)$ & $2.14(0.75)$ & $2.79(0.90)$ \\
High $[969,1069]$ & $1.20(0.11)$ & $1.72(0.16)$ & $1.39(0.09)$ & $2.16(0.25)$ \\
\hline \hline
\end{tabular}

Numbers in brackets represent standard error.

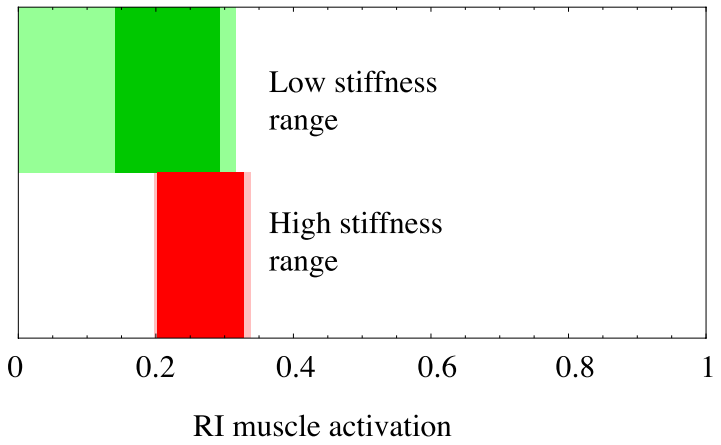

Fig. 6. Biological intrinsic muscle activation data (dark color) overlaid on the available RI solution space (light color) generated using the ACT hand model for a 2-3 N end-effector force. The upper rectangles represent the solution space for low stiffness, and lower rectangles the solution space for high stiffness.

and extrinsic muscles. For example, within an isotorque space, we notice that a range of intrinsic forces is available to vary end-effector stiffness, whereas there is a clear mapping between extrinsic muscle forces and end-effector stiffness-end-effector stiffness increases as extrinsic muscle force increases. Also, in a sensitivity analysis of the muscle forces for a flexion force-stiffness task, the extrinsic muscles have greater sensitivity (less allowed variability) due to their large moment arms.

\section{B. Biological Strategies}

Combining biological data and the ACT hand model, we notice that RI muscle activation (an intrinsic muscle) is high even though low-activation solutions are available. Furthermore, we notice that RI's muscle activation is not controlled closely, since its spread is much larger than the extrinsic muscle activation spread and is large compared to the available solution space (particularly at high-stiffness level).

\section{Contribution of Intrinsic Muscles in Precision Con- trol}

Both extrinsic and intrinsic muscles have large solution spaces (as can be inferred from Fig. 5's scatter and Fig. 6's light shadow) which permit the central nervous system choice in muscle strategies. However, while the central nervous system was selective about the control solution in extrinsic muscle space, it chose to use a significant portion of the intrinsic solution space. This interesting difference between the intrinsic and extrinsic muscle usage could have resulted from different factors. First, the intrinsic muscle space is an uncontrolled manifold and holding a specific solution is not a critical goal for the central nervous system. Another possibility is that the intrinsic muscle is actually heavily used to make fine adjustment of the force and stiffness magnitude and force for the fingertip. There is also a possibility that the intrinsic muscles cannot be controlled as precisely as the extrinsic muscles due to the physiologic/neural structure. We now briefly investigate each possibility.

From biological literature, the last option is not true as it is possible to control intrinsic muscles even more precisely with less motor noise than extrinsic muscles [14]. The intrinsic muscle space has also been shown to be well controlled by our group [1] and others [17], so it is also likely not an uncontrolled manifold. Therefore, we hypothesize that the intrinsic muscle is actually heavily used to make fine adjustment of the force and stiffness magnitude and force for the fingertip. It is similar to having knobs with different gains: If it is important to make fast and large changes in force or stiffness, it is easier to do so using a knob with high gain. And if it is important to make potentially slow but precise quantity change of force/stiffness, it is easier to do so using a knob with smaller gain. Because of the intrinsic muscle's control space structure (see Fig 3), they are able to act as the knobs with smaller gains for precise control. In contrast, the extrinsic muscles which have large moment arms behave as actuators with large control gains. Note that our results are based on the data for only one intrinsic muscle and a more comprehensive validation across multiple intrinsic muscles is required. Finally, we note that increased biological solution variability as actuation increases is expected, since motor noise increases as actuation increases [23], [24].

\section{Implication to Robotics}

First, by studying biological solutions in parallel with robotics models, we infer that redundant actuators with 
low mechanical advantage in a task can still be utilized to improve task stability in addition to providing a choice in solutions. Second, the ACT hand has played a significant role in this work helping estimate the human hand's moment arms. Many biomechanics studies have to approximate human body kinematic parameters, because it is difficult to measure them. Now, the ACT hand offers an alternative for hand research. Since the ACT hand has been built to mimic the details of the human hand, it provides a way to directly estimate various biomechanical parameters. Finally, as future work, while we have previously explored some strategies the central nervous system uses for transitioning between tasks [3], it will be interesting to study path planning in muscle-force space for dynamic movements using the ACT hand framework.

\section{ACKNOWLEDGMENT}

The authors gratefully acknowledge the contribution of Ashish D. Deshpande and Jonathan Ko in running experiments to find the moment arms for the ACT hand. Brian T. Dellon contributed to discussions on control redundancy, and Sameer Agarwal to discussions on constrained linear optimization.

\section{APPENDIX A}

The extrinsic muscles are Extensor Indicis (EI), Flexor Digitorum Profundus (FDP), and Flexor Digitorum Superficialis (FDS), and the intrinsic muscles are Palmar Interosseous (PI), Lumbrical (LUM), and Radial Interosseous (RI).

\section{REFERENCES}

[1] P. Afshar. A Framework for the Neural Control of Limbs with Application to the Index Finger. PhD thesis, Carnegie Mellon University, 2007.

[2] K. N. An, Y. Ueba, E. Y. Chao, W. P. Cooney, and R. I. Linscheid. Tendon excursion and moment arm of index finger muscles. Journal of Biomechanics, 16:419-425, 1983.

[3] R. Balasubramanian and Y. Matsuoka. Biological stiffness control strategies for the Anatomically Correct Testbed (ACT) hand. In Proceedings of the IEEE International Conference on Robotics and Automation, pages 737-742, 2008. DOI 10.1109/ROBOT.2008.4543293.

[4] S. H. M. Brown and S. M. McGill. Muscle force-stiffness characteristics influence joint stability: A spine example. Clinical Biomechanics, 20:917-922, 2005.

[5] L. Y. Chang and Y. Matsuoka. A kinematic thumb model for the act hand. In Proceedings of the 2006 IEEE International Conference on Robotics and Automation, 2006.

[6] J. R. Close and C. C. Kidd. The function of the muscles of the thumb, the index, and long fingers. J Bone Joint Surg, 51, 1969.

[7] C. S. Cook and M. J. N. McDonagh. Measurement of muscle and tendon stiffness in man. Eur J Appl Physiol, 72:380-382, 1996.

[8] J. J. Craig. Introduction to Robotics. Addison Wesley, 1989.
[9] A. D. Deshpande, R. Balasubramanian, R. Lin, B. T. Dellon, and Y. Matsuoka. Understanding variable moment arms for the index finger mcp joints through the act hand. In Proceedings of the IEEE International Conference on Biomedical Robotics and Biomechatronics, 2008.

[10] N. Gialias and Y. Matsuoka. Muscle actuator design for the act hand. In Proceedings of the IEEE International Conference on Robotics and Automation, volume 4, pages 3380-3385, 2004.

[11] J. Hamill and K. M. Knutzen. Biomechanical Basis of Human Movement. Lippincott Williams and Wilkins, 2006.

[12] J. Hoffer and S. Andreassen. Regulation of soleus muscle stiffness in premammillary cats: intrinsic and reflex components. Journal of Neurophysiology, pages 267-285, 1981.

[13] C. A. Klein. Use of redundancy in the design of robotic systems. In H. Hanafusa and H. Inoue, editors, The International Symposium of Robotics Research, 1984.

[14] C. Long, P. W. Conrad, E. A. Hall, and S. L. Furler. Intrinsicextrinsic muscle control of the hand in power grip and precision handling. J Bone Joint Surg, 52, 1970.

[15] M. A. Maier and M. C. Hepp-Reymond. Emg activation during force production in precision grip I: Contribution of fifteen finger muscles to isometric force). Exp Brain Res, 103, 1995.

[16] M. A. Maier and M. C. Hepp-Reymond. Emg activation during force production in precision grip II: Contribution of fifteen finger muscles to isometric force). Exp Brain Res, 103, 1995.

[17] T. E. Milner and S. S. Dhaliwal. Activation of intrinsic and extrinsic finger muscles in relation to the fingertip force vector. Exp. Brain Res., 146:197-204, 2002). DOI: 10.1007/s00221002-1177-7.

[18] T. E. Milner and D. W. Franklin. Characterization of multijoint finger stiffness: dependence on finger posture and force direction. IEEE Trans Biomed Eng, 45:1363-1375, 1998.

[19] Y. Oh, W. K. Chung, Y. Youm, and I. H. Suh. Motion/force decomposition of redundant manipulator and its application to hybrid impedance control. In Proceedings of the IEEE International Conference on Robotics and Automation, volume 2, pages 1441-1446, 1998.

[20] N. S. Pollard and R. C. Gilbert. Tendon arrangement and muscle force requirements for humanlike force capabilities in a robotic finger. In Proceedings of the IEEE International Conference on Robotics and Automation, volume 4, pages 3755-3762, 2002. DOI: 10.1109/ROBOT.2002.1014298.

[21] J. P. Scholz and G. Schöner. The uncontrolled manifold concept: identifying control variables for a functional task. Exp Brain Res, 126(3), 1999. DOI: 10.1007/s002210050738.

[22] R. Shadmehr and M. A. Arbib. A mathematical analysis of the force-stiffness characteristics of muscles in control of a single joint system. Biol. Cybern., 66:463-477, 1992.

[23] G. G. Sutton and K. Sykes. The variation of hand tremor with force in healthy subjects. J. Physiol. Lond., 191:699$711,1967$.

[24] E. Todorov. Cosine tuning minimizes motor errors. Neural Comput., 14:1233-1260, 2002.

[25] F. J. Valero-Cuevas. Predictive modulation of muscle coordination pattern magnitude scales fingertip force magnitude over the voluntary range. J Neurophysiol, 83:1469-1479, 2000.

[26] M. Vande Weghe, M. Rogers, M. Weissert, and Y. Matsuoka. The ACT hand: design of the skeletal structure. In Proceedings of the IEEE International Conference on Robotics and Automation, volume 4, pages 3375-3379, 2004. 